INPLASY

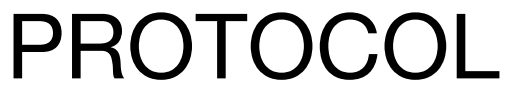

To cite: Guo et al. Risk factors for recurrence of tic disorders in children: a systematic review and Meta-analysis. Inplasy protocol 202220078. doi:

10.37766/inplasy2022.2.0078

Received: 19 February 2022

Published: 19 February 2022

Corresponding author:

Sun Xiang-Juan

hot285@163.com

Author Affiliation:

Hospital of Chengdu

University of Chinese

Medicine.

Support: None.

Review Stage at time of this submission: Piloting of the study selection process.

Conflicts of interest:

None declared.

\section{Risk factors for recurrence of tic disorders in children: a systematic review and Meta-analysis}

Guo, Z1; Yang, K²; Lei, J3; Zeng, Y4; Guo, Z5; Zhang, F6; Sun, X7.

Review question / Objective: P:Children under the age of 18 with tic disorders, no restrictions on gender or ethnicity of participants; I:Presence of a risk factor for recurrence; C:Absence of a risk factor for recurrence; O:Recurrence rate of tic disorders; S:Cohort studies, case-control studies, crosssectional studies.

Condition being studied: Tic disorder (TD) is a neuropsychiatric disorder characterised by sudden, rapid, recurrent, unrhythmic movements or vocalisations that are common in childhood or adolescence. Its pathogenesis and associated causes are unknown, and only a small percentage of children recover spontaneously, while some have recurrent symptoms that persist into adulthood. As tic disorders have a long course and are prone to recurrence, it is crucial to reduce the recurrence of the disorder. Therefore, to further explore the risk factors for recurrence of tic disorder, this study used an evidence-based research method to conduct a meta-analysis of risk factors for recurrence of tic disorder in order to provide an updated evidence-based basis for the prevention and control of recurrence of tic disorder in children.

INPLASY registration number: This protocol was registered with the International Platform of Registered Systematic Review and Meta-Analysis Protocols (INPLASY) on 19 February 2022 and was last updated on 19 February 2022 (registration number INPLASY202220078).

\section{INTRODUCTION}

Review question / Objective: P:Children under the age of 18 with tic disorders, no restrictions on gender or ethnicity of participants; I:Presence of a risk factor for recurrence; $\mathrm{C}$ :Absence of a risk factor for recurrence; O:Recurrence rate of tic disorders; S:Cohort studies, case-control studies, cross-sectional studies.

Condition being studied: Tic disorder (TD) is a neuropsychiatric disorder characterised by sudden, rapid, recurrent, 
unrhythmic movements or vocalisations that are common in childhood or adolescence. Its pathogenesis and associated causes are unknown, and only a small percentage of children recover spontaneously, while some have recurrent symptoms that persist into adulthood. As tic disorders have a long course and are prone to recurrence, it is crucial to reduce the recurrence of the disorder. Therefore, to further explore the risk factors for recurrence of tic disorder, this study used an evidence-based research method to conduct a meta-analysis of risk factors for recurrence of tic disorder in order to provide an updated evidence-based basis for the prevention and control of recurrence of tic disorder in children.

\section{METHODS}

Search strategy: Computer searches of PubMed, EMbase, The Cochrane Library, Web of Science, VIP, WanFang Data and CNKI databases were conducted with a search time frame of from creation to February 2022. The search was conducted using a combination of subject terms and free terms. The search formula for PubMed, for example, is: (("Tic Disorders"[Mesh]) OR (((Tourette disorder[Title/Abstract]) OR (Tourette syndrome[Title/Abstract])) OR (Tourette disorder[Title/Abstract]))) AND (()((()(Recurrence[Title/Abstract]) OR (Recurrences[Title/Abstract])) OR (Recrudescence[Title/Abstract])) OR (Recrudescences[Title/Abstract])) OR (Relapse[Title/Abstract])) OR (Relapses[Title/Abstract])) OR (relapse[Title/Abstract])) OR (risk factors for recurrent[Title/Abstract])).

Participant or population: Children under the age of 18 with Tic disorder, no restrictions on gender or ethnicity of participants.

Intervention: Presence of a risk factor for recurrence.

Comparator: Absence of a risk factor for recurrence.
Study designs to be included: Cohort studies, case-control studies, crosssectional studies.

Eligibility criteria: Inclusion criteria.(i) Participants must be children with tic disorder under the age of 18 years, regardless of gender, geography or ethnicity; (ii) The source of cases was children with confirmed tic disorder in various medical institutions;(iii) The literature should address one or more risk factors for the recurrence of tic disorder; (iv) The study should be a case-control study or cohort study or cross-sectional study;Exclusion criteria.(1) Duplicate published literature;(2) Literature not in Chinese or English;(3)Studies with no control group, incomplete basic data and too many lost visits;(4)Reviews of literature.

Information sources: A comprehensive literature search will be conducted in PubMed, Embase, Cochrane Library, Web of Science, China National Knowledge Infrastructure(CNKI), Wanfang Data and VIP Data, up to February 2022.

Main outcome(s): Recurrence rate of TD.

Quality assessment / Risk of bias analysis: NewcastleOttawa scale (NOS), Agency for Healthcare Research and Qualit (AHRQ) The evaluation scale will be used independently by two researchers. Any problems or disagreements will be resolved through discussion with a third investigator.

Strategy of data synthesis: Statistical analysis was performed using RevMan 5.4 software, and literature that met the inclusion criteria was tested for heterogeneity. If $\mathrm{P}>0.05$ or $\mathrm{I}^{2}<50 \%$ indicated homogeneity between groups, the data were combined using a fixed effects model; if $P<0.05$ or $I^{2} \geq 50 \%$, a random effects model was used and the source of heterogeneity was determined by sensitivity analysis, and descriptive analysis was performed for literature with 
greater heterogeneity. $P<0.05$ for statistically significant difference.

Subgroup analysis: Subgroup analysis was carried out according to the age, gender, place of residence and family background of the children.

Sensitivity analysis: Data from the remaining papers were combined after deleting any one of them in turn, and if the difference between the results before and after deletion was not significant, the sensitivity analysis was considered to have been passed.

Country(ies) involved: China.

Keywords: Risk factors for recurrence, Tic disorders.

Contributions of each author:

Author 1 - Guo Zhiyi.

Author 2 - Yang Kejia.

Author 3 - Lei Jing.

Author 4 - Zeng Yongjian.

Author 5 - Guo Zhidong.

Author 6 - Zhang Fenghua. 